\title{
Concise Total Synthesis of Peyssonnoside A
}

\author{
Chesnokov, Gleb A ; Gademann, Karl
}

\begin{abstract}
Peyssonnoside A is a marine-derived sulfated diterpenoid glucoside with a unique 5/6/3/6 tetracyclic skeleton with a highly substituted cyclopropane ring deeply embedded into the structure. Herein, we report the first total synthesis of this natural product in a concise, efficient, scalable, and highly diastereoselective fashion. The aglucone peyssonnosol was synthesized in $21 \%$ overall yield after 15 steps, featuring a Simmons-Smith cyclopropanation and Mukaiyama hydration, fully controlled by the spatial structure of the substrates.
\end{abstract}

DOI: https://doi.org/10.1021/jacs.1c07135

Posted at the Zurich Open Repository and Archive, University of Zurich ZORA URL: https://doi.org/10.5167/uzh-212596

Journal Article

Published Version

\section{(cc) 980}

The following work is licensed under a Creative Commons: Attribution-NonCommercial-NoDerivatives 4.0 International (CC BY-NC-ND 4.0) License.

Originally published at:

Chesnokov, Gleb A; Gademann, Karl (2021). Concise Total Synthesis of Peyssonnoside A. Journal of the American Chemical Society, 143(35):14083-14088.

DOI: https://doi.org/10.1021/jacs.1c07135 


\title{
Concise Total Synthesis of Peyssonnoside A
}

\author{
Gleb A. Chesnokov and Karl Gademann* \\ Cite This: J. Am. Chem. Soc. 2021, 143, 14083-14088 \\ Read Online
}

ABSTRACT: Peyssonnoside A is a marine-derived sulfated diterpenoid glucoside with a unique 5/6/3/6 tetracyclic skeleton with a highly substituted cyclopropane ring deeply embedded into the structure. Herein, we report the first total synthesis of this natural product in a concise, efficient, scalable, and highly diastereoselective fashion. The aglucone peyssonnosol was synthesized in $21 \%$ overall yield after 15 steps, featuring a Simmons-Smith cyclopropanation and Mukaiyama hydration, fully controlled by the spatial structure of the substrates.

$\mathrm{M}$ arine natural products are of great importance and interest in modern chemistry due to the broad scope of biological activity and structural diversity they provide. ${ }^{1,2}$ In particular, polycyclic strained structures containing small rings, such as, e.g., cyclopropanes, constitute synthetically challenging targets. ${ }^{3}$ To date there are only a few examples of polycyclic natural products with densely substituted cyclopropanes being successfully synthesized. ${ }^{4-8}$

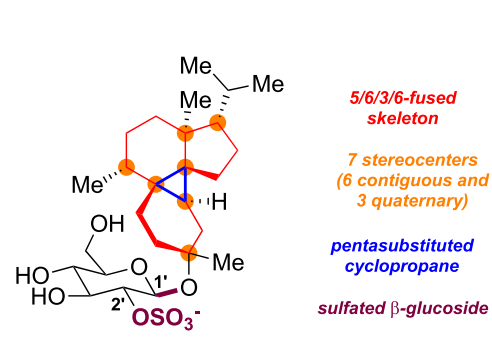

Peyssonnoside A (1)

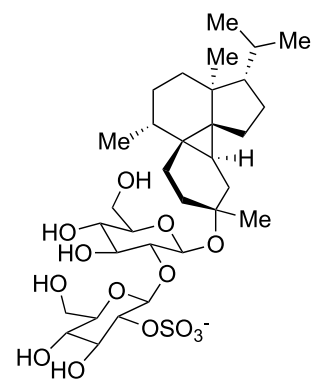

Peyssonnoside B (2)
In 2019, Kubanek and co-workers ${ }^{9}$ reported the isolation of two marine sulfated diterpene glucosides, peyssonnosides $\mathrm{A}$ (1) and B (2), from the red alga Peyssonnelia sp. sharing the same diterpenoid core with a highly substituted and sterically encumbered cyclopropane ring, which were shown to have promising activity against liver stage Plasmodium berghei and moderate antimethicillin-resistant Staphylococcus aureus (MRSA) activity. The unusual and complicated structure combined with the diverse biological activity render peyssonnosides challenging yet promising molecular targets for synthetic organic chemists (Scheme 1). Salient features of the peyssonnoside diterpenoid core include (1) a unique and unprecedented tetracyclic 5/6/3/6 carbon framework with a pentasubstituted cyclopropane ring deeply embedded into the structure (Scheme 1); (2) seven stereogenic centers out of which six are contiguous; (3) three quaternary contiguous stereocenters; and (4) a pentasubstituted cyclopropane ring. In addition, the sulfated $\beta$-linked glycoside poses an additional challenge due to the tertiary nature of the hydroxy group in the
Scheme 1. Retrosynthetic Analysis
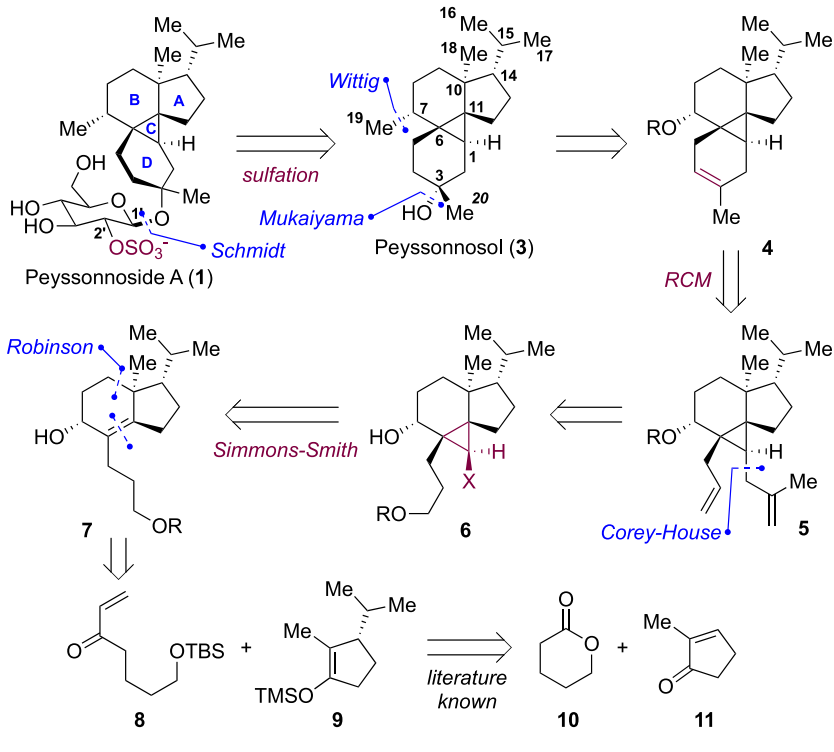

aglucone 3, which can lead to elimination and orthoester byproducts instead.

Our retrosynthetic analysis started with the search for the most appropriate approach to tackle the cyclopropane moiety. ${ }^{10}$ Functional modification of the aglucone 3 (peyssonnosol, Scheme 1) to 4 provides a useful intermediate accessible via ring-closing metathesis (RCM). Further disconnection led to cyclopropane 6 via a postulated $\mathrm{Cu}$ mediated Corey-House reaction. ${ }^{11}$ In turn, 6 was assumed to be accessible via Simmons-Smith cyclopropanation, ${ }^{12,13}$ leading to the intermediate 7 , which in turn can be traced

Received: July 13, 2021

Published: August 25, 2021 
back to precursors $8^{14}$ and $9^{15}$ known in the literature via Robinson annulation. ${ }^{16}$

The required starting materials were prepared according to procedures known from the literature: 9 from 2-methyl-2cyclopenten-1-one (11) in 1 step in $95 \%$ yield $^{15}$ and $\mathbf{8}$ from $\gamma$ valerolactone (10) in 3 steps (one pot) in 50\% overall yield (Scheme 2). ${ }^{14}$ Mukaiyama-type Michael addition of 9 to 8

Scheme 2. Synthesis of Peyssonnosol (3)
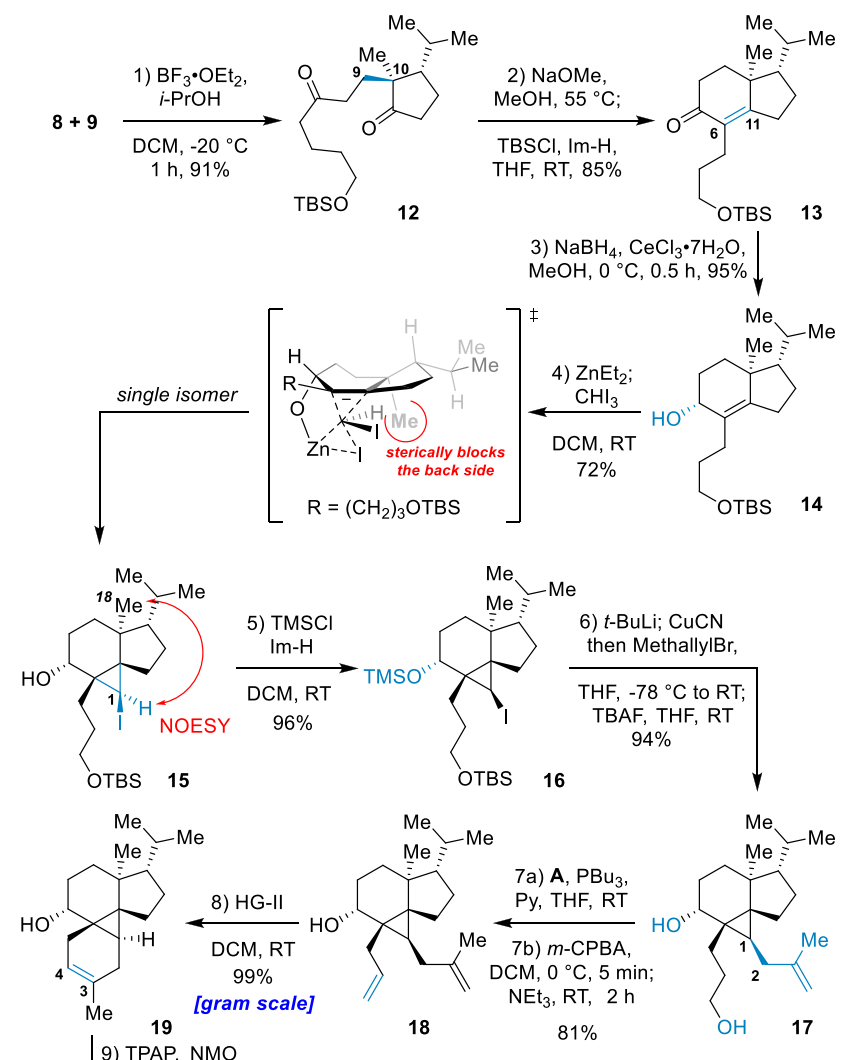

DCM, RT, $99 \%$

$\checkmark$

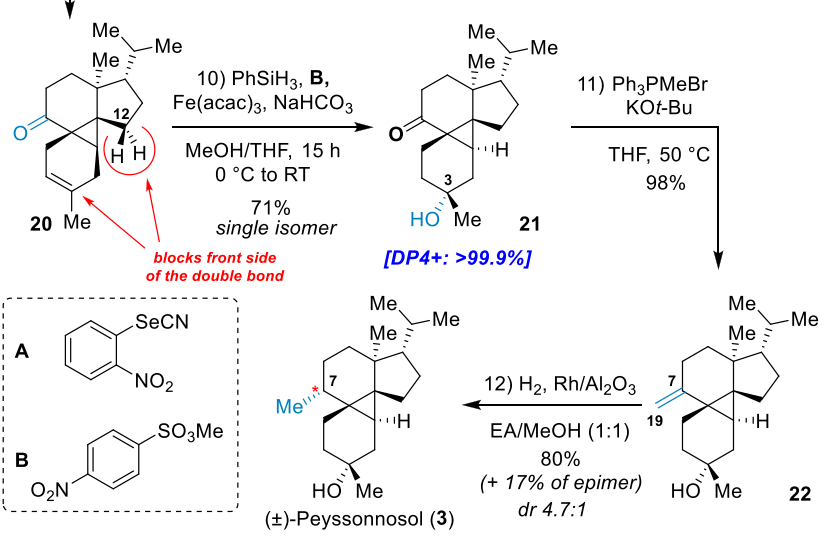

(士)-Peyssonnosol (3)

furnished diketone $\mathbf{1 2}$ via diastereoselective formation of the $\mathrm{C}(9)-\mathrm{C}(10)$ in $91 \%$ yield as a single diastereoisomer as observed by NMR spectroscopy. The reaction was performed under Lewis acid catalysis in a $\mathrm{BF}_{3} \cdot \mathrm{Et}_{2} \mathrm{O} / i$ - $\mathrm{PrOH}$ system at $-20{ }^{\circ} \mathrm{C}$, initially developed by Poirier and co-workers. ${ }^{17}$ Then, diketone 12 was treated with an excess of $\mathrm{NaOMe}$ in $\mathrm{MeOH}$ at $55{ }^{\circ} \mathrm{C}$ to complete the Robinson annulation sequence and the construction of the $\mathbf{B}$ ring. ${ }^{16}$ To account for the partial desilylation during the cyclization step, the intermediate cyclized product was treated with $\mathrm{TBSCl} / \mathrm{Im}-\mathrm{H}$, providing the bicyclic enone 13 in $85 \%$ yield. This enone was further diastereoselectively reduced under Luche conditions to afford allylic alcohol 14 in 95\% yield, via an exclusive pseudoequatorial attack of the carbonyl group by the borohydride species. ${ }^{18-20}$ This intermediate set the stage for a challenging step in our synthetic route, the Simmons-Smith cyclopropanation of a tetrasubstituted double bond with a monosubstituted carbenoid. Fortunately, we were delighted to find that the original conditions developed by Furukawa and co-workers ${ }^{21}$ performed with iodoform delivered the desired product 14 in $55 \%$ yield as a single diastereoisomer, thus successfully furnishing the cyclopropane $\mathbf{C}$ ring construction. A strong cross-peak between protons at $\mathrm{C}(1)$ and $\mathrm{C}(18)$ in NOESY experiments provided excellent evidence for the assigned configuration. Lowering the addition temperature to $0{ }^{\circ} \mathrm{C}$ allowed an increase in the yield up to $72 \%$. Further investigation of this step demonstrated that the addition order of the reagents is of paramount importance for the success of the transformation. Treating the alcohol first with diethylzinc ensured the formation of mixed zinc alkyl-alkoxide species, further improving the directing group ability of the alkoxide. Subsequent addition of the iodoform solution presumably led to the formation of a $\mathrm{Zn}$ carbenoid species bonded directly to the alkoxide and thus close to the double bond, which is hypothesized to be key to the success of this reaction. Of central importance to note was that only one out of four possible diastereoisomers was observed. The syn-reaction (to $\mathrm{OH}$ ) of the cyclopropane is guided by the alkoxide group directing effect, which moreover allowed for the energetically more stable cis-A,B-ring formation. The exo-I installation can be attributed to the steric effect of the axial methyl group $\mathrm{C}(18)$, blocking the interior of the down side of the molecule. Overall, Simmons-Smith cyclopropanation led to the successful installation of three stereocenters in one step.

The obtained iodocyclopropane $\mathbf{1 5}$ was further protected with $\mathrm{TMSCl} / \mathrm{Im}-\mathrm{H}$ for the subsequent Corey-House crosscoupling. ${ }^{22,23} \mathrm{Cu}$-mediated $\mathrm{sp}^{3}-\mathrm{sp}^{3}$ coupling was experimentally assessed to be more robust and reliable when compared to conventional cross-coupling catalysts. ${ }^{24-26}$ Iodocyclopropane 16 was first lithiated with $t$-BuLi, and the thus formed lithium species was treated with the $\mathrm{Cu}$ source to form an organocopper intermediate, which was further reacted with methallyl bromide. Among the different copper sources $[\mathrm{Li}(2-$ $\mathrm{Th}) \mathrm{CuCN}, \mathrm{CuCN}, \mathrm{CuI}, \mathrm{CuSCN}$, and $\mathrm{CuSPh}$ ] evaluated, $\mathrm{CuCN}$ was experimentally established to be the most efficient, providing the product in $94 \%$ yield after desilylation of the intermediate with TBAF, cleanly furnishing the $\mathrm{C}(1)-\mathrm{C}(2)$ bond formation. The obtained enediol 17 was further submitted to the Grieco dehydration protocol ${ }^{27,28}$ in order to convert the primary $\mathrm{RCH}_{2} \mathrm{CH}_{2} \mathrm{OH}$ functionality of $\mathbf{1 7}$ to the corresponding vinyl group. The formation of the corresponding 2-nitrophenylselenoether was selective and efficient (92\%). However, the subsequent elimination step presented experimental challenges; typical procedures for the oxidation of the substrate with an excess of $\mathrm{H}_{2} \mathrm{O}_{2}$ in DCM or THF were found to be slow, irreproducible, and prone to epoxidation of the terminal olefin of the methallyl side chain. Subsequent experimentation identified the addition of 1.0 equiv of $m$-CPBA at $0{ }^{\circ} \mathrm{C}$ as crucial for clean oxidation in less than $5 \mathrm{~min}$, and subsequent addition of $\mathrm{NEt}_{3}$ with stirring at room temperature (RT) for $2 \mathrm{~h}$ afforded the corresponding dienol 18 in $81 \%$ yield (over the two steps). RCM of dienol 18 with $1 \mathrm{~mol} \%$ of second-generation Hoveyda-Grubbs 
catalyst $^{29,30}$ furnished the quantitative formation of the $\mathbf{D}$ cycle in 19 in less than $4 \mathrm{~h}$ at RT on a gram scale. Thus, at this point, we achieved a robust, efficient, and scalable construction of the unique tetracyclic $5 / 6 / 3 / 6$ backbone of the peyssonnosides in only 8 steps in $38 \%$ overall yield, which enables routes to structural derivatives that can be used for biological investigations.

With the tetracyclic skeleton of the target diterpenoid prepared, the next steps included the functional group modification in order to install all required functionalities in peyssonnosol (3). First, the alcohol 19 was quantitatively converted to the corresponding ketone (20) via Ley-Griffith oxidation $^{31}$ with TPAP/NMO. It is noteworthy that ketone $\mathbf{2 0}$ provides an interesting example of the anisotropic magnetic effect of the carbonyl group: the methylene protons at $\mathrm{C}(5)$ display a $1.55 \mathrm{ppm}$ difference in chemical shifts. With the ketone 20 in hand, the Mukaiyama hydration protocol ${ }^{32}$ was attempted; however, no conversion was observed. At the time of these failed attempts, an anaerobic Mukaiyama hydration protocol with a large scope of terpenoids was published by Studer and co-workers. ${ }^{33}$ This method involved the use of nitroarylsulfonate $\mathbf{B}$ and Fe catalysis, which led to the tertiary alcohol 21 in good yield (71\%). The hydration afforded only one diastereoisomer 21, which was assigned to be the desired one based on 2D NMR experiments and computation of DP4+ probability $(>99.9 \%) .{ }^{34}$ The high facial selectivity could be explained with structural rigidity of the ketone 20, with the $\mathrm{C}(12)$ methylene group effectively blocking one side of the double bond from the attack, which gives an interesting example of exclusive diastereoselectivity governed by molecule topology. ${ }^{35}$ The obtained ketoalcohol 21 was further submitted to Wittig methylenation under standard conditions $^{36}\left(\mathrm{Ph}_{3} \mathrm{PMeBr} / \mathrm{KO} t-\mathrm{Bu}\right)$ affording the corresponding unsaturated alcohol $\mathbf{2 2}$ in $98 \%$ yield. The diastereoselectivity of the hydrogenation of compound $\mathbf{2 2}$ proved to be highly dependent on both the catalyst and solvent ranging from 1:6.8 for $\left(\mathrm{Ph}_{3} \mathrm{P}\right)_{3} \mathrm{RhCl}$ in toluene to $4.7: 1$ for $\mathrm{Rh} / \mathrm{Al}_{2} \mathrm{O}_{3}$ in $\mathrm{EA} /$ $\mathrm{MeOH}$ (1:1); however, the conversion was almost always quantitative. Fortunately, the diastereisomeric mixture can be easily separated by standard flash column chromatography. Thus, with the installation of the $\mathrm{C}(7)$ hydrogen atom, our synthesis of racemic peyssonnosol (3), the tetracyclic 5/6/3/6 diterpenoid core of peyssonnosides $\mathrm{A}-\mathrm{B}(\mathbf{1 - 2})$, was accomplished. It was achieved in only 12 steps from a compound known from the literature and in $21 \%$ overall yield with high diastereoselectivity. The developed route is scalable, and more than $0.5 \mathrm{~g}$ of 3 was prepared in a single run.

With 3 in hand, we moved to the glucosylation stage of synthesis (Scheme 3). As a glucosyl donor, we favored the trichloroacetimidates introduced by Schmidt and co-workers ${ }^{37}$ due to the mild activation conditions needed for the reaction. ${ }^{38,39}$ In order to be able to install sulfate functionality at $\mathrm{C}\left(2^{\prime}\right)$, this position should be orthogonally protected from the other hydroxy groups of the glucosyl donor. The best match for our purposes was the compound 23, easily accessible from D-glucose in 7 steps. ${ }^{40}$ With the glucoside donor in hand, we attempted the glucosylation. However, it turned out that typical conditions (TMSOTf, ${ }^{41} \mathrm{BF}_{3} \cdot \mathrm{Et}_{2} \mathrm{O}^{39}$ ) led to the complete decomposition of peyssonnosol (3) (most probably, through dehydration), which was associated with high acid sensitivity of the peyssonnosol (3). As a consequence, it was hypothesized that other frequently used activators (Ln(OTf) ${ }_{3}{ }^{42,43} \mathrm{Tf}_{2} \mathrm{O},{ }^{44}$ etc.) would not work due to their high
Scheme 3. Completion of the Synthesis

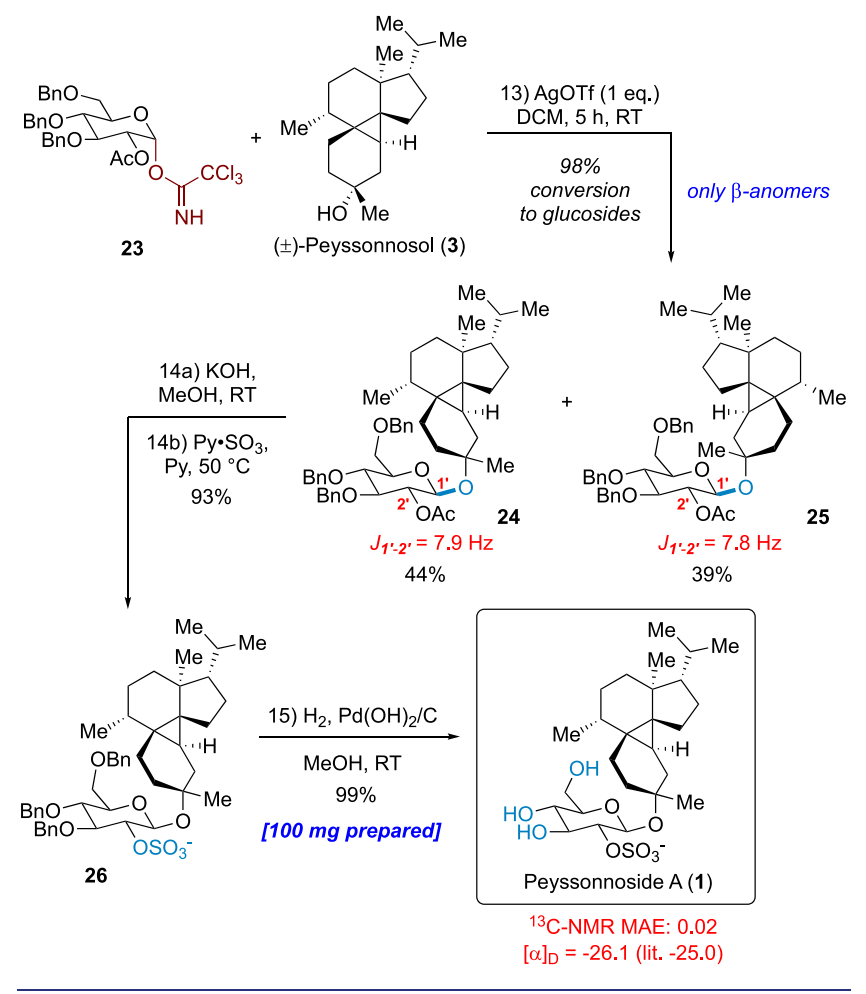

oxophilicity, and in our case, more nitrophilic activators are needed. For that role, three candidates were evaluated $\left(\mathrm{Cu}(\mathrm{OTf})_{2},{ }^{45} \mathrm{AgOTf}^{46}\right.$ and $\left.(\mathrm{PhCN})_{2} \mathrm{Pd}(\mathrm{OTf})_{2}{ }^{47}\right)$, and AgOTf led to the best performance. After some optimization runs, 3 equiv of the glucoside donor 23 and 1 equiv of AgOTf in DCM at RT after $5 \mathrm{~h}$ provided $98 \%$ yield of the $1: 1$ diastereomeric mixture of glucosides of both peyssonnosol (3) enantiomers (24 and 25). This mixture can be separated by flash column chromatography on silica gel with $\mathrm{CHCl}_{3}$. The less polar component was identified to be the desired one based on the comparison between the relative positions of proton signals at $\mathrm{C}(18)$ and $\mathrm{C}(1)$ in ${ }^{1} \mathrm{H}$ NMR spectra and the corresponding data from the isolation study, ${ }^{9}$ assuming that further peripheral modification would not change the core geometry. Isolated yields were $88 \%$ and $78 \%$, respectively, based on both enantiomers of 3 . Based on J-coupling constant analysis of protons at $\mathrm{C}\left(1^{\prime}\right)$ and $\mathrm{C}\left(2^{\prime}\right)(7.9 \mathrm{~Hz}$ for 24 and 7.8 $\mathrm{Hz}$ for 25$)$, both glucosides were assigned as pure $\beta$-isomers. The diastereoisomer $\mathbf{2 4}$ was further hydrolyzed with $\mathrm{KOH} /$ $\mathrm{MeOH}$ and sulfated on the hydroxy group without intermediate purification affording the sulfate $\mathbf{2 6}$ in $93 \%$ yield. Hydrogenolysis of the benzyl groups over Pearlman's catalyst afforded peyssonnoside A (1) in 99\% yield. All characterization data $\left({ }^{1} \mathrm{H}\right.$ and ${ }^{13} \mathrm{C}$ NMR, HRMS, $\left.[\alpha]_{\mathrm{D}}\right)$ matched those reported for the isolated natural product. Therefore, we achieved the first total synthesis of enantiopure peyssonnoside A in only 15 steps.

The synthesis can be optimized further by an enantioselective approach to peyssonnosol (3, Scheme 4) with the asymmetric 1,4-addition step to $\mathbf{1 1}$ as a key challenge. In general, this chemistry is well-developed, ${ }^{48}$ yet 2 -methyl-2cyclopenten-1-one (11) turned out to be a quite challenging substrate, for which it is difficult to achieve high enantioselectivity. ${ }^{49-51}$ Quinkert and co-workers ${ }^{52}$ reported in 1992 that isopropenyl lithium can be asymmetrically added 
Scheme 4. Enantioselective Approach to (-)-Peyssonnosol (5)

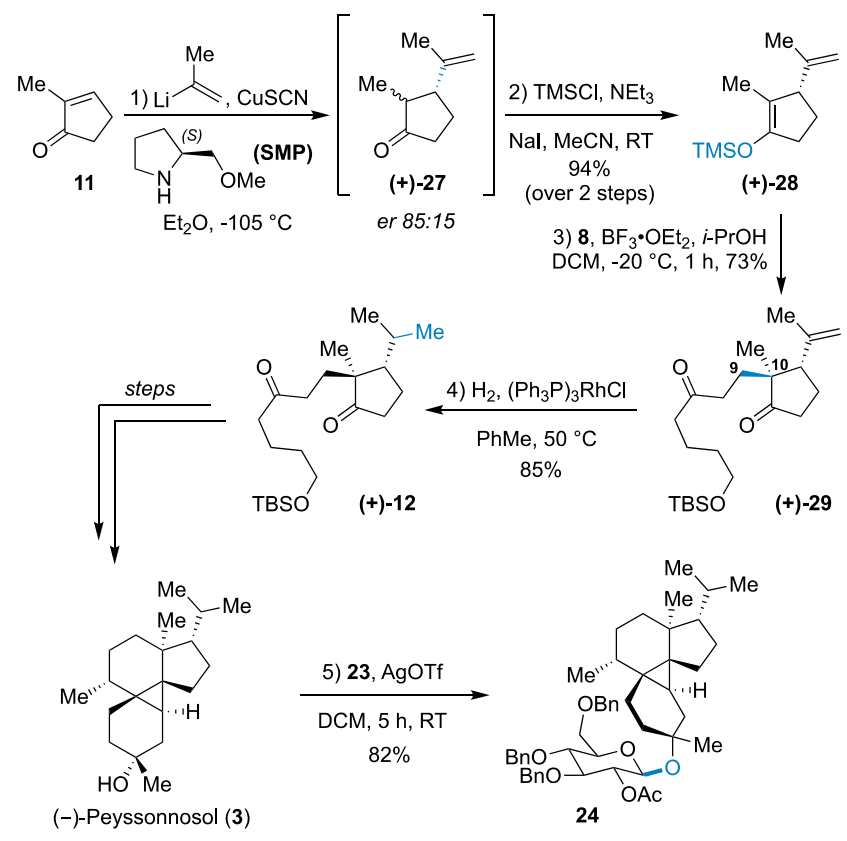

to 11 in the presence of excess of $O$-methyl-D-prolinol (SMP) affording the desired $(R)$-enantiomer of 27 with $60-89 \%$ ee, which was used in a $(+)$-confertin synthesis. ${ }^{53}$ This method was adopted for our synthesis. Consequently, the product of the asymmetric 1,4-addition (+)-27, obtained in 85:15 er, was first converted to the corresponding silyl enol ether (+)-28, which was further submitted to the Mukaiyama-type Michael addition step, as described above. The product (+)-29 was then hydrogenated with Wilkinson's catalyst to give (+)-12 in $85 \%$ yield. This product was converted to $(-)$-peyssonnosol (3) with the sequence described above. (-)-Peyssonnosol (3) was also glucosylated and gave the desired diastereomer 24 in $82 \%$ yield, further confirming the absolute configuration of 27 as shown.

In conclusion, we have achieved the first total synthesis of peyssonnoside A (1). The natural product was prepared in synthetic form in 15 steps with its diterpenol core [peyssonnosol (3)] prepared in only 12 steps from literatureknown compound $\mathbf{8}$, which is accessible in a 3 step one pot fashion from a commercial starting material. Key features of the synthesis include (1) construction of the $\mathbf{B}$ ring via Robinson annulation, (2) installation of the pentasubstituted cyclopropane $\mathbf{C}$ ring via highly diastereoselective SimmonsSmith cyclopropanation with iodoform, (3) construction of the $\mathrm{D}$ ring via RCM of the diene $\mathbf{1 8}$ successfully obtained through Corey-House cross-coupling, (4) installation of the isolated stereocenter at $\mathrm{C}(3)$ with a highly diastereoselective anaerobic Mukaiyama hydration, and (5) efficient and $\beta$-selective Schmidt glucosylation with subsequent sulfation of the hydroxy group at $\mathrm{C}\left(2^{\prime}\right)$. All 7 stereocenters were installed in a highly diastereoselective fashion, harnessing the intrinsic substrate bias. The synthetic route was also modified to allow access to the preparation of the enantiopure core. For the first time, the synthesis paves the way to the 5/6/3/6 tetracyclic skeleton with a pentasubstituted cyclopropane ring.

\section{ASSOCIATED CONTENT}

\section{Supporting Information}

The Supporting Information is available free of charge at https://pubs.acs.org/doi/10.1021/jacs.1c07135.

Experimental procedures and copies of NMR spectra (PDF)

Coordinate files for conformers of $\mathbf{2 1}$ and its C3-epimer (ZIP)

\section{AUTHOR INFORMATION}

\section{Corresponding Author}

Karl Gademann - Department of Chemistry, University of Zurich, CH-8057 Zurich, Switzerland; orcid.org/00000003-3053-0689; Email: karl.gademann@uzh.ch

\section{Author \\ Gleb A. Chesnokov - Department of Chemistry, University of Zurich, CH-8057 Zurich, Switzerland}

Complete contact information is available at:

https://pubs.acs.org/10.1021/jacs.1c07135

\section{Funding}

We thank the Swiss National Science Foundation for financial support of this work (182043).

Notes

The authors declare no competing financial interest.

\section{ACKNOWLEDGMENTS}

We thank Marco Schellenberg for help with early prospecting investigations en route to 3 as part of his undergraduate studies and Kevin Keller for technical assistance.

\section{REFERENCES}

(1) Carroll, A. R.; Copp, B. R.; Davis, R. A.; Keyzers, R. A.; Prinsep, M. R. Marine natural products. Nat. Prod. Rep. 2021, 38 (2), 362413.

(2) Carroll, A. R.; Copp, B. R.; Davis, R. A.; Keyzers, R. A.; Prinsep, M. R. Marine natural products. Nat. Prod. Rep. 2020, 37 (2), 175223.

(3) Fan, Y.-Y.; Gao, X.-H.; Yue, J.-M. Attractive natural products with strained cyclopropane and/or cyclobutane ring systems. Sci. China: Chem. 2016, 59 (9), 1126-1141.

(4) Miller, A. K.; Trauner, D. Total Synthesis of ( \pm )-Photodeoxytridachione through a Lewis Acid Catalyzed Cyclization. Angew. Chem., Int. Ed. 2003, 42 (5), 549-552.

(5) Jin, S.; Gong, J.; Qin, Y. Total Synthesis of (-)-Lundurine A and Determination of its Absolute Configuration. Angew. Chem., Int. Ed. 2015, 54 (7), 2228-2231.

(6) Levin, S.; Nani, R. R.; Reisman, S. E. Enantioselective Total Synthesis of (+)-Salvileucalin B. J. Am. Chem. Soc. 2011, 133 (4), 774-776.

(7) Kirillova, M. S.; Muratore, M. E.; Dorel, R.; Echavarren, A. M. Concise Total Synthesis of Lundurines A-C Enabled by Gold Catalysis and a Homodienyl Retro-Ene/Ene Isomerization. J. Am. Chem. Soc. 2016, 138 (11), 3671-3674.

(8) Xu, W.; Zhao, J.; Tao, C.; Wang, H.; Li, Y.; Cheng, B.; Zhai, H. Collective Total Synthesis of (-)-Lundurines A-C. Org. Lett. 2018, 20 (6), 1509-1512.

(9) Khatri Chhetri, B.; Lavoie, S.; Sweeney-Jones, A. M.; Mojib, N.; Raghavan, V.; Gagaring, K.; Dale, B.; McNamara, C. W.; Soapi, K.; Quave, C. L.; Polavarapu, P. L.; Kubanek, J. Peyssonnosides A-B, Unusual Diterpene Glycosides with a Sterically Encumbered Cyclopropane Motif: Structure Elucidation Using an Integrated Spectro- 
scopic and Computational Workflow. J. Org. Chem. 2019, 84 (13), $8531-8541$.

(10) Ebner, C.; Carreira, E. M. Cyclopropanation Strategies in Recent Total Syntheses. Chem. Rev. 2017, 117 (18), 11651-11679.

(11) Knochel, P.; Harenberg, J. H. The Corey-House Reaction: An Early Transition-Metal-Mediated Cross-Coupling. Synfacts 2020, 16 (04), No. 0437.

(12) Beaulieu, L.-P. B.; Zimmer, L. E.; Charette, A. B. Enantio- and Diastereoselective Iodocyclopropanation of Allylic Alcohols by Using a Substituted Zinc Carbenoid. Chem. - Eur. J. 2009, 15 (44), 1182911832.

(13) Kim, H. Y.; Lurain, A. E.; García-García, P.; Carroll, P. J.; Walsh, P. J. Enantio- and Diastereoselective Tandem Preparation of Cyclopropyl Alcohols. Synfacts 2005, 2005 (03), 0330-0330.

(14) Labarre-Lainé, J.; Beniazza, R.; Desvergnes, V.; Landais, Y. Convergent Access to Bis-spiroacetals through a Sila-StetterKetalization Cascade. Org. Lett. 2013, 15 (18), 4706-4709.

(15) Piers, E.; Renaud, J. Total synthesis of the tetraquinane diterpenoid ( \pm -crinipellin B. J. Org. Chem. 1993, 58 (1), 11-13.

(16) Duhamel, P.; Dujardin, G.; Hennequin, L.; Poirier, J.-M. Lewis acid catalysed Michael-type addition. A new regio- and diastereoselective annulation method using methyl vinyl ketone. J. Chem. Soc., Perkin Trans. 1 1992, No. 3, 387-396.

(17) Poirier, J.-M.; Hennequin, L. A new bis-annelation method. Application to steroid synthesis. Tetrahedron 1989, 45 (13), 41914202.

(18) Luche, J. L. Lanthanides in organic chemistry. 1. Selective 1,2 reductions of conjugated ketones. J. Am. Chem. Soc. 1978, 100 (7), 2226-2227.

(19) Luche, J.-L.; Rodriguez-Hahn, L.; Crabbé, P. Reduction of natural enones in the presence of cerium trichloride. J. Chem. Soc., Chem. Commun. 1978, 0 (14), 601-602.

(20) Nagasawa, K.; Matsuda, N.; Noguchi, Y.; Yamanashi, M.; Zako, Y.; Shimizu, I. Stereoselective synthesis of cyclopentanones by reductive cleavage of 6-oxonorbornane-2-carboxylates and its application to the synthesis of $1 \alpha, 25$-dihydroxyvitamin $\mathrm{D} 3 \mathrm{CD}$ ring. J. Org. Chem. 1993, 58 (6), 1483-1490.

(21) Furukawa, J.; Kawabata, N.; Nishimura, J. A stereospecific synthesis of cyclopropane derivatives from olefins. Tetrahedron Lett. 1968, 9 (31), 3495-3498.

(22) Corey, E. J.; Posner, G. H. Selective formation of carboncarbon bonds between unlike groups using organocopper reagents. J. Am. Chem. Soc. 1967, 89 (15), 3911-3912.

(23) House, H. O.; Respess, W. L.; Whitesides, G. M. The Chemistry of Carbanions. XII. The Role of Copper in the Conjugate Addition of Organometallic Reagents1. J. Org. Chem. 1966, 31 (10), 3128-3141.

(24) Allouche, E. M. D.; Taillemaud, S.; Charette, A. B. Spectroscopic characterization of (diiodomethyl)zinc iodide: application to the stereoselective synthesis and functionalization of iodocyclopropanes. Chem. Commun. 2017, 53 (69), 9606-9609.

(25) Beaulieu, L.-P. B.; Zimmer, L. E.; Gagnon, A.; Charette, A. B. Highly Enantioselective Synthesis of 1,2,3-Substituted Cyclopropanes by Using $\alpha$-Iodo- and $\alpha$-Chloromethylzinc Carbenoids. Chem. - Eur. J. 2012, 18 (46), 14784-14791.

(26) Kim, H. Y.; Salvi, L.; Carroll, P. J.; Walsh, P. J. Highly Enantioand Diastereoselective One-Pot Methods for the Synthesis of Halocyclopropyl Alcohols. J. Am. Chem. Soc. 2009, 131 (3), 954-962.

(27) Grieco, P. A.; Gilman, S.; Nishizawa, M. Organoselenium chemistry. A facile one-step synthesis of alkyl aryl selenides from alcohols. J. Org. Chem. 1976, 41 (8), 1485-1486.

(28) Sharpless, K. B.; Young, M. W. Olefin synthesis. Rate enhancement of the elimination of alkyl aryl selenoxides by electron-withdrawing substituents. J. Org. Chem. 1975, 40 (7), 947949.

(29) Garber, S. B.; Kingsbury, J. S.; Gray, B. L.; Hoveyda, A. H. Efficient and Recyclable Monomeric and Dendritic Ru-Based Metathesis Catalysts. J. Am. Chem. Soc. 2000, 122 (34), 8168-8179.
(30) Gessler, S.; Randl, S.; Blechert, S. Synthesis and metathesis reactions of a phosphine-free dihydroimidazole carbene ruthenium complex. Tetrahedron Lett. 2000, 41 (51), 9973-9976.

(31) Ley, S. V.; Norman, J.; Griffith, W. P.; Marsden, S. P. Tetrapropylammonium Perruthenate, $\mathrm{Pr}_{4} \mathrm{~N}^{+} \mathrm{RuO}_{4}^{-}$, TPAP: A Catalytic Oxidant for Organic Synthesis. Synthesis 1994, 1994 (07), 639666.

(32) Isayama, S.; Mukaiyama, T. A New Method for Preparation of Alcohols from Olefins with Molecular Oxygen and Phenylsilane by the Use of Bis(acetylacetonato)cobalt(II). Chem. Lett. 1989, 18 (6), 1071-1074.

(33) Bhunia, A.; Bergander, K.; Daniliuc, C. G.; Studer, A. FeCatalyzed Anaerobic Mukaiyama-Type Hydration of Alkenes using Nitroarenes. Angew. Chem., Int. Ed. 2021, 60 (15), 8313-8320.

(34) Grimblat, N.; Zanardi, M. M.; Sarotti, A. M. Beyond DP4: an Improved Probability for the Stereochemical Assignment of Isomeric Compounds using Quantum Chemical Calculations of NMR Shifts. J. Org. Chem. 2015, 80 (24), 12526-12534.

(35) Hu, P.; Chi, H. M.; DeBacker, K. C.; Gong, X.; Keim, J. H.; Hsu, I. T.; Snyder, S. A. Quaternary-centre-guided synthesis of complex polycyclic terpenes. Nature 2019, 569 (7758), 703-707.

(36) (a) Fitjer, L.; Quabeck, U. The Wittig Reaction Using Potassium-tert-butoxide High Yield Methylenations of Sterically Hindered Ketones. Synth. Commun. 1985, 15 (10), 855-864. For recent applications, see (b) Rosenbaum, L.-C; Häfner, M.; Gaich, T. Total Synthesis of the Diterpene Waihoensene. Angew. Chem., Int. Ed. 2021, 60, 2939-2942. (c) Peng, C.; Arya, P.; Zhou, Z.; Snyder, S. A. A Concise Total Synthesis of (+)-Waihoensene Guided by Quaternary Center Analysis. Angew. Chem., Int. Ed. 2020, 59, $13521-13525$.

(37) Schmidt, R. R.; Michel, J. Facile Synthesis of $\alpha$ - and $\beta$-OGlycosyl Imidates; Preparation of Glycosides and Disaccharides. Angew. Chem., Int. Ed. Engl. 1980, 19 (9), 731-732.

(38) Yang, Y.; Zhang, X.; Yu, B. O-Glycosylation methods in the total synthesis of complex natural glycosides. Nat. Prod. Rep. 2015, 32 (9), 1331-1355.

(39) Das, R.; Mukhopadhyay, B. Chemical O-Glycosylations: An Overview. ChemistryOpen 2016, 5 (5), 401-433.

(40) Loscher, S.; Schobert, R. Total Synthesis and Absolute Configuration of Epicoccamide D, a Naturally Occurring Mannosylated 3-Acyltetramic Acid. Chem. - Eur. J. 2013, 19 (32), 1061910624.

(41) Schaubach, R.; Hemberger, J.; Kinzy, W. Tumor-associated antigen synthesis synthesis of the gal- $\alpha-(1 \rightarrow 3)$-gal- $\beta-(1 \rightarrow 4)$-GlcNAc epitope a specific determinant for metastatic progression? Liebigs Ann. Chem. 1991, 1991 (7), 607-614.

(42) Adinolfi, M.; Iadonisi, A.; Ravidà, A. Tunable Activation of Glycosyl Trichloro- and (N-phenyl)trifluoro-acetimidates with Ytterbium(III) Triflate: One-Pot Synthesis of Trisaccharides under Catalytic Conditions. Synlett 2006, 2006 (04), 0583-0586.

(43) Adinolfi, M.; Barone, G.; Guariniello, L.; Iadonisi, A. Efficient activation of armed glycosyl trichloroacetimidates with $\mathrm{Sm}(\mathrm{OTf})_{3}$ in the stereoselective glycosidation of saccharidic acceptors. Tetrahedron Lett. 2000, 41 (46), 9005-9008.

(44) Dobarro-Rodriguez, A.; Trumtel, M.; Wessel, H. P. Triflic Anhydride: An Alternative Promoter in Glycosidations. J. Carbohydr. Chem. 1992, 11 (3), 255-263.

(45) Yamada, H.; Hayashi, T. A substrate-unspecified glycosylation reaction promoted by copper(II) trifluoromethanesulfonate in benzotrifluoride. Carbohydr. Res. 2002, 337 (7), 581-585.

(46) Douglas, S. P.; Whitfield, D. M.; Krepinsky, J. J. Silver Trifluoromethanesulfonate(Triflate) Activation of Trichloroacetimidates in Glycosylation Reactions. J. Carbohydr. Chem. 1993, 12 (1), 131-136.

(47) Mensah, E. A. Palladium and nickel catalyzed stereoselective formation of glycosides. $\mathrm{PhD}$ Dissertation. University of Iowa, Iowa City, IA, 2011. DOI: 10.17077/etd.3wencq01 (accessed 2021-08-19).

(48) Vargová, D.; Némethová, I.; Šebesta, R. Asymmetric coppercatalyzed conjugate additions of organometallic reagents in the 
syntheses of natural compounds and pharmaceuticals. Org. Biomol. Chem. 2020, 18 (20), 3780-3796.

(49) Calvo, B. C.; Madduri, A. V. R.; Harutyunyan, S. R.; Minnaard, A. J. Copper-Catalysed Conjugate Addition of Grignard Reagents to 2-Methylcyclopentenone and Sequential Enolate Alkylation. Adv. Synth. Catal. 2014, 356 (9), 2061-2069.

(50) Germain, N.; Alexakis, A. Formation of Contiguous Quaternary and Tertiary Stereocenters by Sequential Asymmetric Conjugate Addition of Grignard Reagents to 2-Substituted Enones and $\mathrm{Mg}-$ Enolate Trapping. Chem. - Eur. J. 2015, 21 (23), 8597-8606.

(51) Germain, N.; Guénée, L.; Mauduit, M.; Alexakis, A. Asymmetric Conjugate Addition to $\alpha$-Substituted Enones/Enolate Trapping. Org. Lett. 2014, 16 (1), 118-121.

(52) Quinkert, G.; Müller, T.; Königer, A.; Schultheis, O.; Sickenberger, B.; Dürner, G. Enantioselective Conjugate Addition Greatly Improves the Synthesis of (+)-Confertin. Tetrahedron Lett. 1992, 33 (24), 3469-3472.

(53) Quinkert, G.; Schmalz, H.-G.; Walzer, E.; KowalczykPrzewloka, T.; Düner, G.; Bats, J. W. Total Synthesis of the Pseudoguaianolide (+)-Confertin. Angew. Chem., Int. Ed. Engl. 1987, 26 (1), 61-62. 